\title{
DoA Estimation Using Low-Resolution Multi-Bit Sparse Array Measurements
}

\author{
Saeid Sedighi, Student Member, IEEE, M. R. Bhavani Shankar, Senior Member, IEEE, Mojtaba \\ Soltanalian, Senior Member, IEEE, and Björn Ottersten, Fellow, IEEE
}

\begin{abstract}
This letter studies the problem of Direction of Arrival (DoA) estimation from low-resolution few-bit quantized data collected by Sparse Linear Array (SLA). In such cases, contrary to the one-bit quantization case, the well known arcsine law cannot be employed to estimate the covaraince matrix of unquantized array data. Instead, we develop a novel optimizationbased framework for retrieving the covaraince matrix of unquantized array data from low-resolution few-bit measurements. The MUSIC algorithm is then applied to an augmented version of the recovered covariance matrix to find the source DoAs. The simulation results show that increasing the sampling resolution to 2 or 4 bits per samples could significantly increase the DoA estimation performance compared to the one-bit sampling regime while the power consumption and implementation costs is still much lower in comparison to the high-resolution sampling implementations.
\end{abstract}

Index Terms-Direction of arrival (DoA) estimation, lowresolution quantization, Sparse linear arrays, few-bit quantization.

\section{INTRODUCTION}

Direction of Arrival (DoA) estimation from Uniform Linear Array (ULA) measurements is extensively studied in the literature [1-3]. However, the number of identifiable sources with ULAs is limited to the number of array elements minus one [3, 4]. Deployment of Sparse Linear Arrays (SLAs), e.g. Minimum Redundancy Arrays (MRAs) [5], co-prime arrays [6] and nested arrays [7], allows for transcending this limitation under the assumption of uncorrelated source signals such that the number of identifiable sources can go considerably beyond the number array elements. A detailed study on the performance of DoA estimation via SLAs has been conducted in [8] through an analysis of the Cramér-Rao Bound (CRB). Further, a variety of algorithms for estimating DoAs from SLA data have been presented in the literature [7, 9, 15].

Most of the algorithms developed for estimating DoAs from SLA measurements are based on the assumption that quantization errors are negligible as a result of using high-resolution

This work was partially supported by the Luxembourg National Research Fund (FNR) under the ACCORDION project (Ref: 11228830), the SPRINGER the European Research Council (ERC) Grant AGNOSTIC (ID: 742648), U.S National Science Foundation (NSF) Grants 1704401, 1809225, and an Illinois Discovery Partners Institute (DPI) Seed Award.

S. Sedighi, M. R. B. Shankar and B. Ottersten are with the Interdisciplinary Centre for Security, Reliability and Trust (SnT), University of Luxembourg, Luxembourg City L-1855, Luxembourg (e-mails: saeid.sedighi@uni.lu; bhavani.shankar@uni.lu; bjorn.ottersten@uni.lu). M. Soltanalian is with the Department of Electrical and Computer Engineering, University of Illinois at Chicago, Chicago, IL 60607 USA (e-mail: msol@uic.edu).
Analog-to-Digital Converters (ADCs). However, use of highresolution ADCs is typically expensive and power-hungry [16]. Hence, to reduce energy consumption and production costs, DoA estimation with binary measurements collected by onebit ADCs has been recently proposed and discussed in the literature [17,-27]. One-bit ADCs represent each sample of the analog array observations with only a single bit offering, an exceedingly high sampling rate at a low production cost and very low power consumption [16]. The analytical performance bounds for DoA estimation from one-bit data have been studied in [28-30]. Further, a number of one-bit DoA estimators have been provided in [23,-25, 27], which rest on retrieving the covariance matrix of unquantized array observations using the well-known Bussgang theorem [31].

In this paper, as opposed to the previous works which have studied the problem of DoA estimation under two extreme scenarios for analog-to-digital conversion, i.e., infinite-bit quantization and one-bit quantization, we aim to investigate the problem of estimating DoAs from low-resolution few-bit SLA measurements. In such cases, contrary to the one-bit quantization case, the Bussgang theorem may not be directly employed to retrieve the covaraince matrix of array unquantized observations. Instead, we develop a novel optimization-based framework for retrieving the covaraince matrix of unquantized array observations from low-resolution multi-bit measurements. Then, we apply the Co-Array-Based MUSIC (CAB-MUSIC) [7, 13] to the recovered covariance matrix to find the DoAs of interest. The simulation results show that increasing the sampling resolution with a few bits per samples could significantly improve the DoA estimation performance compared to the one-bit sampling case while the power consumption and implementation costs are still much lower than the highresolution scenario.

Paper organization: The system model is described in Section II Section III presents the proposed algorithm for estimating DoAs from few-bit data. Simulation results are shown and discussed in Section IV. Finally, conclusions are drawn in Section V

Notation: : Lightface, lower- and upper-case bold-face letters denote scalars, vectors and matrices, respectively. The conjugate, transpose and Hermitian (conjugate transpose) operations are referred to by the superscripts $*, T, H$, respectively. $\|\mathbf{A}\|_{F}$ and $\operatorname{rank}(\mathbf{A})$ stand for the Frobenius norm and the rank of $\mathbf{A}$, respectively. $[\mathbf{a}]_{i}$ indicates the $i^{\text {th }}$ entry of $\mathbf{a}$. $\operatorname{diag}(\mathbf{a})$ is a diagonal matrix made out of entries of a. $\mathbf{I}_{M}$ denotes an $M \times M$ identity matrix. $\Re\{a\}$ and $\Im\{a\}$ stand for the real and imaginary parts of $a$, respectively. 
(a)

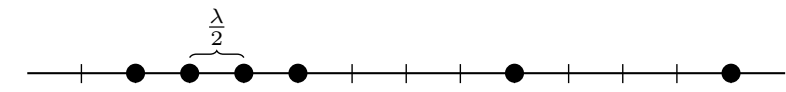

(b)

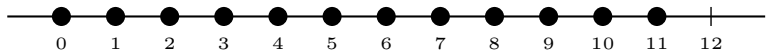

Figure 1. (a) An SLA with $\mathbb{M}=\{1,2,3,4,8,12\}$; (b) corresponding difference co-array with $\mathbb{D}=\{0,1, \cdots, 11\}$.

\section{SySTEM MODEL}

We consider an SLA with $M$ elements located at positions $m_{1} \frac{\lambda}{2}, m_{2} \frac{\lambda}{2}, \cdots, m_{M} \frac{\lambda}{2}$ with $m_{i} \in \mathbb{M}$. Here $\lambda$ denotes the wavelength of the incoming signals and $\mathrm{M}$ is a set of integers with a cardinality of $M$. It is assumed that $K$ narrowband signals with distinct DoAs $\boldsymbol{\theta}=\left[\theta_{1}, \theta_{2}, \cdots, \theta_{K}\right]^{T}$ impinge on the SLA from far-field. The signal received by the array at time instance $t$ can be modeled as

$$
\mathbf{y}(t)=\mathbf{A}(\boldsymbol{\theta}) \mathbf{s}(t)+\mathbf{n}(t) \in \mathbb{C}^{M \times 1}, \quad t=0, \cdots, N-1,
$$

where $\mathbf{s}(t) \in \mathbb{C}^{K \times 1}$ denotes the vector of $K$ source signals, $\mathbf{n}(t) \in \mathbb{C}^{M \times 1}$ is additive noise, and $\mathbf{A}(\boldsymbol{\theta})=$ $\left[\mathbf{a}\left(\theta_{1}\right), \mathbf{a}\left(\theta_{2}\right), \cdots, \mathbf{a}\left(\theta_{K}\right)\right] \in \mathbb{C}^{M \times K}$ represents the SLA steering matrix with

$$
\mathbf{a}\left(\theta_{k}\right)=\left[e^{j \pi \sin \theta_{k} m_{1}}, e^{j \pi \sin \theta_{k} m_{2}}, \cdots, e^{j \pi \sin \theta_{k} m_{M}}\right]^{T},
$$

being the SLA manifold vector for the $k^{\text {th }}$ signal. Further, the following assumptions are made on source and noise signals:

A1 $\mathbf{n}(t)$ follows a zero-mean circular complex Gaussian distribution with the covariance matrix $\mathbb{E}\left\{\mathbf{n}(t) \mathbf{n}^{H}(t)\right\}=\sigma^{2} \mathbf{I}_{M}$.

A2 The source signals are modeled as zero-mean uncorrelated circular complex Gaussian random variables with covariance matrix $\mathbb{E}\left\{\mathbf{s}(t) \mathbf{s}^{H}(t)\right\}=\operatorname{diag}(\mathbf{p})$ where $\mathbf{p}=\left[p_{1}, p_{2}, \cdots, p_{K}\right]^{T} \in \mathbb{R}_{>0}^{K \times 1}$ (i.e., $p_{k}>0, \forall k$ ).

A3 No temporal correlation is assumed between the snapshots, i.e., $\mathbb{E}\left\{\mathbf{n}\left(t_{1}\right) \mathbf{n}^{H}\left(t_{2}\right)\right\}=\mathbb{E}\left\{\mathbf{s}\left(t_{1}\right) \mathbf{s}^{H}\left(t_{2}\right)\right\}=\mathbf{0}$ when $t_{1} \neq$ $t_{2}$ and $\mathbf{0}$ is an all-zero matrix of appropriate dimensions. Based on the above assumptions, the covariance matrix of $\mathbf{y}(t)$ is given by

$$
\mathbf{R}=\mathbb{E}\left\{\mathbf{y}(t) \mathbf{y}^{H}(t)\right\}=\mathbf{A}(\boldsymbol{\theta}) \operatorname{diag}(\mathbf{p}) \mathbf{A}^{H}(\boldsymbol{\theta})+\sigma^{2} \mathbf{I}_{M} \in \mathbb{C}^{M \times M} .
$$

It is readily verified that $\mathbf{R}$ is a structured matrix with only $2 D-1$ free parameters where $D=|\mathbb{D}|$ with $\mathbb{D}=\left\{\left|m_{p}-m_{q}\right|\right.$ : $\left.m_{p}, m_{q} \in \mathbb{M}\right\}$. The set $\mathbb{D}$ is called the difference co-array [ 8 . 15]. Noticing the structure in $\mathbf{R}$, it can be rewritten as follows

$$
\mathbf{R}(\mathbf{u})=u_{0} \mathbf{L}_{0}+\sum_{n=1}^{D-1} u_{n} \mathbf{L}_{n}+\sum_{n=1}^{D-1} u_{n}^{*} \mathbf{L}_{n}^{T},
$$

where $u_{0}=\sigma^{2}+\sum_{k=1}^{K} p_{k}, u_{n}=\sum_{k=1}^{K} p_{k} e^{j \pi \sin \theta_{k} \ell_{n}} \quad$ and

$$
\left[\mathbf{L}_{n}\right]_{p, q}=\left\{\begin{array}{lc}
1, & \text { if } m_{p}-m_{q}=\ell_{n} \\
0, & \text { otherwise }
\end{array}\right.
$$

with $\ell_{n} \in \mathbb{D}, m_{p}, m_{q} \in \mathbb{M}, 1 \leq p, q \leq M$ and $0 \leq n \leq$ $D-1$. A proper design of SLA allows for identifying more uncorrelated source signals than the number of array elements by exploiting the resulting structure of $\mathbf{R}$ efficiently [6, 8,15$]$. Fig. 1 illustrates an SLA along with its difference co-array.

In the classical mode, the received signals are sampled at Nyquist rate and processed assuming full-precision analog-todigital conversion. On the other hand, herein, we assume that each array sensor is equipped with a low-resolution multi-bit ADC converting the received analog signal into digital data using $q$ bits per sample. A generic $q$-bit ADC has $2^{q}-1$ threshold levels where $\alpha_{1}<\alpha_{2}<\cdots<0<\cdots<\alpha_{2 q}<\alpha_{2 q}-1$. The $q$-bit ADC at the $m^{\text {th }}$ array element transforms the real and imaginary parts of $[\mathbf{y}(t)]_{m}$ into one of the $2^{q}-1$ prescribed qunatization levels $\left\{\gamma_{1}, \gamma_{2}, \cdots, \gamma_{2^{q}-1}\right\}$ by comparing them individually with the threshold levels. Particularly, the $q$-bit quantized output signal at the $m^{\text {th }}$ array element is expressed as

$$
[\mathbf{x}(t)]_{m}=Q\left([\Re\{\mathbf{y}(t)\}]_{m}\right)+j Q\left([\Im\{\mathbf{y}(t)\}]_{m}\right),
$$

where $Q($.$) denotes the q$-bit quantization operation defined as

$$
Q(a)=\gamma_{h} \quad \text { if } \quad \alpha_{h} \leq a<\alpha_{h+1} .
$$

We are interested in estimating DoAs from $q$ bit quantized output signals of the SLA, i.e., $\mathbf{X}=[\mathbf{x}(0), \quad \mathbf{x}(1), \quad \cdots, \quad \mathbf{x}(N-1)]$.

\section{Multi-Bit DoA estimation with Sparse Arrays}

In this section, we first formulate an optimization problem whose solution provides us with an estimate of the covariance matrix of $\mathbf{y}(t)$, i.e., $\mathbf{R}$, using $q$-bit quantized array measurements, i.e., X. Subsequently, we apply the CAB-MUSIC [7] 13] to obtain DoA estimates from the estimate of $\mathbf{R}$.

It follows from (4) that $\mathbf{R}$ is fully described by the complex vector $\mathbf{u}=\left[u_{0}, u_{1}, \cdots, u_{D-1}\right]^{T}$. Hence, for a given $\mathbf{Y}=$ $[\mathbf{y}(0), \mathbf{y}(1), \cdots, \quad \mathbf{y}(N-1)], \mathbf{R}$ can be obtained from the solution of the following optimization problem

$$
\begin{array}{ll}
\underset{\mathbf{u}}{\operatorname{minimize}} & \left\|\mathbf{R}(\mathbf{u})-\mathbf{Y} \mathbf{Y}^{H}\right\|^{2} \\
\text { subject to } & \mathbf{R}(\mathbf{u}) \succeq \mathbf{0} .
\end{array}
$$

However, $\mathbf{Y}$ is unknown here, and instead, we only have access to its $q$-bit quantized values, i.e., $\mathbf{X}$. It follows from (6) and (7) that each element of the observation matrix $\mathbf{X}$ determines a lower and an upper bound for the real and imaginary parts of the corresponding element in $\mathbf{Y}$. Putting these lower and upper bound into the matrices $\Gamma_{l}$ and $\boldsymbol{\Gamma}_{u}$, an optimization problem for joint estimation of $\mathbf{u}$ and $\mathbf{Y}$ can be cast as follows:

$$
\begin{array}{ll}
\underset{\mathbf{u}, \mathbf{Y}}{\operatorname{minimize}} & \left\|\mathbf{R}(\mathbf{u})-\mathbf{Y} \mathbf{Y}^{H}\right\|_{F}^{2} \\
\text { subject to } & \mathbf{R}(\mathbf{u}) \succeq \mathbf{0}, \\
& \operatorname{vec}\left(\Re\{\mathbf{Y}\}-\Re\left\{\boldsymbol{\Gamma}_{l}\right\}\right) \geq \mathbf{0}, \\
& \operatorname{vec}\left(\Im\{\mathbf{Y}\}-\Im\left\{\boldsymbol{\Gamma}_{l}\right\}\right) \geq \mathbf{0}, \\
& -\left[\operatorname{vec}\left(\Re\{\mathbf{Y}\}-\Re\left\{\boldsymbol{\Gamma}_{u}\right\}\right)\right] \geq \mathbf{0}, \\
& -\left[\operatorname{vec}\left(\Im\{\mathbf{Y}\}-\Im\left\{\boldsymbol{\Gamma}_{u}\right\}\right)\right] \geq \mathbf{0} .
\end{array}
$$

where the last four constraints in 9) aim to enforce the consistency of $\mathbf{Y}$ with the $q$-bit measurements by ensuring that the elements of $\mathbf{Y}$ lie in the regions determined by the observation matrix $\mathbf{X}$. The above optimization problem is nonconvex as its objective is a quartic function with respect to $\mathbf{Y}$. In what follows, we first present an equivalent reformulation for (9), which paves the way for iteratively solving this non-convex optimization problem.

Theorem 1. Consider slack variables $\mathbf{G} \in \mathbb{C}^{(M+N) \times M}$, W $\in$ $\mathbb{C}^{M \times M}$ and $\xi \in \mathbb{R}$. The optimization problem $(9)$ is equivalent to

$$
\begin{array}{cl}
\begin{array}{c}
\text { minimize } \\
\mathbf{u}, \mathbf{W}, \mathbf{G}, \xi
\end{array} & \|\mathbf{R}(\mathbf{u})-\mathbf{W}\|_{F}^{2}+\eta \xi \\
\text { subject to } & \mathbf{R}(\mathbf{u}) \succeq \mathbf{0}, \\
& \operatorname{vec}\left(\Re\{\mathbf{Y}\}-\Re\left\{\boldsymbol{\Gamma}_{l}\right\}\right) \geq \mathbf{0}, \\
& \operatorname{vec}\left(\Im\{\mathbf{Y}\}-\Im\left\{\boldsymbol{\Gamma}_{l}\right\}\right) \geq \mathbf{0}, \\
& -\left[\operatorname{vec}\left(\Re\{\mathbf{Y}\}-\Re\left\{\boldsymbol{\Gamma}_{u}\right\}\right)\right] \geq \mathbf{0}, \\
& -\left[\operatorname{vec}\left(\Im\{\mathbf{Y}\}-\Im\left\{\boldsymbol{\Gamma}_{u}\right\}\right)\right] \geq \mathbf{0}, \\
& \mathbf{T} \succeq \mathbf{0}, \\
& \xi \mathbf{I}_{M}-\mathbf{G}^{H} \mathbf{T G} \succeq \mathbf{0}, \\
& \mathbf{G}^{H} \mathbf{G}=\mathbf{I}_{M},
\end{array}
$$


where $\mathbf{T}=\left[\begin{array}{cc}\mathbf{I}_{N} & \mathbf{Y}^{H} \\ \mathbf{Y} & \mathbf{W}\end{array}\right] \in \mathbb{C}^{(M+N) \times(M+N)}$ and $\eta$ is a regularization parameter.

Proof. Consider the slack variables $\mathbf{W}=\mathbf{Y} \mathbf{Y}^{H}$. Then it is readily seen that the optimization problem (9) is equivalent to:

$$
\begin{array}{cl}
\underset{\mathbf{u}, \mathbf{Y}, \mathbf{W}}{\operatorname{minimize}} & \|\mathbf{R}(\mathbf{u})-\mathbf{W}\|_{F}^{2} \\
\text { subject to } & \mathbf{R}(\mathbf{u}) \succeq \mathbf{0}, \\
& \operatorname{vec}\left(\Re\{\mathbf{Y}\}-\Re\left\{\boldsymbol{\Gamma}_{l}\right\}\right) \geq \mathbf{0}, \\
& \operatorname{vec}\left(\Im\{\mathbf{Y}\}-\Im\left\{\boldsymbol{\Gamma}_{l}\right\}\right) \geq \mathbf{0}, \\
& -\left[\operatorname{vec}\left(\Re\{\mathbf{Y}\}-\Re\left\{\boldsymbol{\Gamma}_{u}\right\}\right)\right] \geq \mathbf{0}, \\
& -\left[\operatorname{vec}\left(\Im\{\mathbf{Y}\}-\Im\left\{\boldsymbol{\Gamma}_{u}\right\}\right)\right] \geq \mathbf{0}, \\
& \mathbf{W}=\mathbf{Y} \mathbf{Y}^{H} .
\end{array}
$$

It is readily confirmed that $\mathbf{W}=\mathbf{Y} \mathbf{Y}^{H}$ if and only if $\operatorname{rank}\left(\mathbf{W}-\mathbf{Y} \mathbf{Y}^{H}\right)=0$. Further, $\operatorname{rank}\left(\mathbf{W}-\mathbf{Y} \mathbf{Y}^{H}\right)=0$ can be equivalently expressed as $\operatorname{rank}\left(\mathbf{I}_{N}\right)+\operatorname{rank}\left(\mathbf{W}-\mathbf{Y} \mathbf{Y}^{H}\right)=N$. Since $\mathbf{I}_{N}$ is positive definite, it follows from the Guttman rank additivity formula [32] that $\operatorname{rank}\left(\mathbf{I}_{N}\right)+\operatorname{rank}\left(\mathbf{W}-\mathbf{Y} \mathbf{Y}^{H}\right)=$ $\operatorname{rank}(\mathbf{T})$. Moreover, it follows from $\mathbf{W}-\mathbf{Y} \mathbf{Y}^{H}=\mathbf{0}$ and $\mathbf{I}_{N} \succ \mathbf{0}$ that $\mathbf{T}$ has to be positive semi-definite. These imply that the equality constraint in (11) can be replaced with a rank constraint on a semi-definite matrix. Hence, the optimization problem (11), and equivalently (9), can be recast as follows:

$$
\begin{array}{cl}
\underset{\mathbf{u}, \mathbf{Y}, \mathbf{W}}{\operatorname{minimize}} & \|\mathbf{R}(\mathbf{u})-\mathbf{W}\|^{2} \\
\text { subject to } & \mathbf{R}(\mathbf{u}) \succeq \mathbf{0}, \\
& \operatorname{vec}\left(\Re\{\mathbf{Y}\}-\Re\left\{\boldsymbol{\Gamma}_{l}\right\}\right) \geq \mathbf{0}, \\
& \operatorname{vec}\left(\Im\{\mathbf{Y}\}-\Im\left\{\boldsymbol{\Gamma}_{l}\right\}\right) \geq \mathbf{0}, \\
& -\left[\operatorname{vec}\left(\Re\{\mathbf{Y}\}-\Re\left\{\boldsymbol{\Gamma}_{u}\right\}\right)\right] \geq \mathbf{0}, \\
& -\left[\operatorname{vec}\left(\Im\{\mathbf{Y}\}-\Im\left\{\boldsymbol{\Gamma}_{u}\right\}\right)\right] \geq \mathbf{0}, \\
& \mathbf{T} \succeq \mathbf{0}, \\
& \operatorname{rank}(\mathbf{T})=N .
\end{array}
$$

The constraint $\operatorname{rank}(\mathbf{T})=N$ in (12) is equivalent to imposing the constraint that the $M$ smallest eigenvalues of $\mathbf{T}$ are all zero. This constraint on the $M$ smallest eigenvalues of $\mathbf{T}$ can be formulated by introducing the new slack variables $\mathbf{G} \in \mathbb{C}^{(M+N) \times M}$ and $\xi \in \mathbb{R}$ with $\mathbf{G}^{H} \mathbf{G}=\mathbf{I}_{M}$. Indeed, in what follows, we will show the $M$ smallest eigenvalues of $T$ are all zero if $\xi \mathbf{I}_{M}-\mathbf{G}^{H} \mathbf{T G} \succeq \mathbf{0}$ and $\xi \rightarrow 0$. Let $\rho_{1} \leq \rho_{2} \leq$ $\cdots \leq \rho_{M+N}$ and $\nu_{1} \leq \nu_{2} \leq \cdots \leq \nu_{M}$ denote the eigenvalues of $\mathbf{T}$ and $\mathbf{G}^{H} \mathbf{T G}$, respectively. From $\xi \mathbf{I}_{M}-\mathbf{G}^{H} \mathbf{T G} \succeq \mathbf{0}$, we have $\nu_{i} \leq \xi$ for $i=1,2, \cdots, M$. Additionally, it follows from [33, Corollary 4.3.16] that $0 \leq \rho_{i} \leq \nu_{i}$ for $i=1,2, \cdots, M$. Hence, we observe that

$$
\mathbf{0} \preceq \operatorname{diag}\left(\left[\rho_{1}, \rho_{2}, \cdots, \rho_{M}\right]^{T}\right) \preceq \operatorname{diag}\left(\left[\nu_{1}, \nu_{2}, \cdots, \nu_{M}\right]^{T}\right) \preceq \xi \mathbf{I}_{M} .
$$

It easily observed from (13) that $\xi \rightarrow 0$ leads the $M$ smallest eigenvalues of $\mathbf{T}$ to go to zero. Accordingly, we can deduce that, by properly selecting $\eta$ in (13) such that the optimum value of $\xi$ goes to zero, the constraints $\xi \mathbf{I}_{M}-\mathbf{G}^{H} \mathbf{T G} \succeq \mathbf{0}$ and $\mathbf{G}^{H} \mathbf{G}=\mathbf{I}_{M}$ in (13) will be equivalent to the rank constraint in (12). This implies that (13) is equivalent to $(12)$ and thus to 9). This completes the proof.

The optimization problem (10) can be solved iteratively by alternating between $\mathbf{G}$ and the other parameters, i.e., $\mathbf{u}, \mathbf{Y}$, $\mathbf{W}$ and $\xi$. Let $\mathbf{G}^{(k)}, \mathbf{u}^{(k)}, \mathbf{Y}^{(k)}, \mathbf{W}^{(k)}$ and $\xi^{(k)}$ be the values of the parameters $\mathbf{G}, \mathbf{u}, \mathbf{Y}, \mathbf{W}$ and $\xi$ at the $k$-th iteration, respectively. Given $\mathbf{G}^{(k-1)}$, the optimization problem with respect to $\mathbf{u}, \mathbf{Y}, \mathbf{W}$ and $\xi$ at the $k$-th iteration becomes

$$
\begin{array}{cl}
\underset{\mathbf{u}^{(k)}, \mathbf{Y}^{(k)}, \mathbf{W}^{(k)}, \xi^{(k)}}{\operatorname{minimize}} & \left\|\mathbf{R}\left(\mathbf{u}^{(k)}\right)-\mathbf{W}^{(k)}\right\|_{F}^{2}+\eta \xi^{(k)} \\
\text { subject to } & \mathbf{R}\left(\mathbf{u}^{(k)}\right) \succeq \mathbf{0}, \\
& \operatorname{vec}\left(\Re\left\{\mathbf{Y}^{(k)}\right\}-\Re\left\{\boldsymbol{\Gamma}_{l}\right\}\right) \geq \mathbf{0}, \\
& \operatorname{vec}\left(\Im\left\{\mathbf{Y}^{(k)}\right\}-\Im\left\{\boldsymbol{\Gamma}_{l}\right\}\right) \geq \mathbf{0}, \\
& -\left[\operatorname{vec}\left(\Re\left\{\mathbf{Y}^{(k)}\right\}-\Re\left\{\boldsymbol{\Gamma}_{u}\right\}\right)\right] \geq \mathbf{0}, \\
& -\left[\operatorname{vec}\left(\Im\left\{\mathbf{Y}^{(k)}\right\}-\Im\left\{\boldsymbol{\Gamma}_{u}\right\}\right)\right] \geq \mathbf{0}, \\
& \mathbf{T}^{(k)} \succeq \mathbf{0}, \\
& \xi^{(k)} \mathbf{I}_{M}-\mathbf{G}^{(k-1)}{ }^{H} \mathbf{T}^{(k)} \mathbf{G}^{(k-1)} \succeq \mathbf{0}, \\
& \xi^{(k)} \leq \xi^{(k-1)} .
\end{array}
$$

Once $\mathbf{T}^{(k)}, \mathbf{u}^{(k)}$ and $\xi^{(k)}$ are found by solving (14), $\mathbf{G}^{(k)}$ can be obtained by seeking an $(M+N) \times M$ matrix with orthonormal columns such that $\mathbf{G}^{(k)}{ }^{H} \mathbf{T}^{(k)} \mathbf{G}^{(k)} \preceq \xi^{(k)} \mathbf{I}_{M}$. Choosing $\mathbf{G}^{(k)}$ to be equal to the matrix composed of the eigenvectors of $\mathbf{T}^{(k)}$ corresponding to its $M$ smallest eigenvalues, and following similar arguments provided after (12), we have

$$
\begin{aligned}
& \mathbf{G}^{(k)^{H}} \mathbf{T}^{(k)} \mathbf{G}^{(k)}=\operatorname{diag}\left(\left[\rho_{1}^{(k)}, \rho_{2}^{(k)}, \cdots, \rho_{M}^{(k)}\right]^{T}\right) \\
& \preceq \operatorname{diag}\left(\left[\nu_{1}^{(k-1)}, \nu_{2}^{(k-1)}, \cdots, \nu_{M}^{(k-1)}\right]^{T}\right) \preceq \xi^{(k)} \mathbf{I}_{M},
\end{aligned}
$$

where $\rho_{1}^{(k)} \leq \rho_{2}^{(k)} \leq \cdots \leq \rho_{M+N}^{(k)}$ and $\nu_{1}^{(k-1)} \leq$ $\nu_{2}^{(k-1)} \leq \cdots \leq \nu_{M}^{(k-1)}$ denote the eigenvalues of $\mathbf{T}^{(k)}$ and $\mathbf{G}^{(k-1)}{ }^{\bar{H}} \mathbf{T}^{(k)} \mathbf{G}^{(k-1)}$, respectively. It follows from 15 that the matrix composed of the eigenvectors of $\mathbf{T}^{(k)}$ corresponding to its $M$ smallest eigenvalue is a right choice of $\mathbf{G}^{(k)}$. Accordingly, at each iteration of the proposed algorithm, we need to solve a Semi-Definite Program (SDP), which can be solved efficiently, followed by an Eigenvalue Decomposition (ED). The alternating optimization procedure is repeated until either the objective or the optimization variables converge to a constant value. Algorithm 1 summarizes the steps of the aforementioned iterative approach to solving (9). Further, to initialize the algorithm, $\mathbf{G}^{(0)}$ can be found through the ED of $\mathbf{T}^{(0)}$ obtained from solving (12) without considering the rank constraint. We note that the proposed algorithm, which is based on alternating optimization method, is guaranteed to converge to at least a local minimum of (10) [34]. Once $\mathbf{R}$ is retrieved from Algorithm 1] the CAB-MUSIC [7, 13] is applied to the retrieved $\mathbf{R}$ to estimate DoAs.

\section{Simulation Results}

In this section, numerical results are provided for assessing the performance of the proposed algorithm for estimating DoAs from low-resolution few-bit SLA output. In all experiments, each simulated point has been computed by 1000 Monte Carlo repetitions over noise realizations. In addition, the $K$ independent sources with an equal power $p$ are equispaced in the angular domain $\left[-60^{\circ}, 60^{\circ}\right]$ with respect to a 8 -sensor nested array with $\mathbb{I M}:\{1,2,3,4,5,10,15,20\}$. The SNR is also defined as $10 \log \frac{p}{\sigma^{2}}$.

Fig. 2 depicts the Root-Mean-Squares-Error (RMSE) for $\theta_{2}$ in degree versus SNR for different bit-width when $N=300$, $M=8$ and: (a) $K=4<M$; (b) $K=10>M$. Fig. 2 demonstrates that increasing the number of quantization bits from one to two and then to four leads to a considerable performance improvement. Further, it is observed that the RMSE of 4-bit 

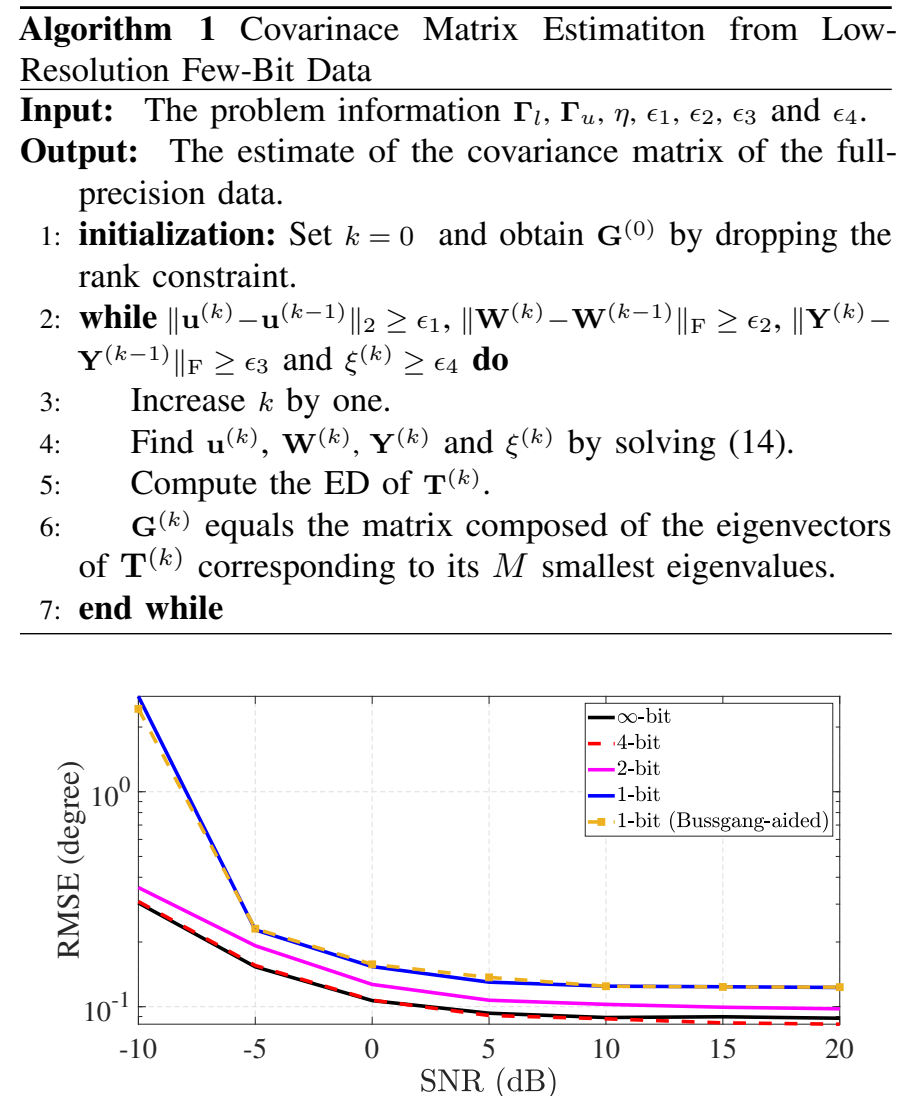

(a)

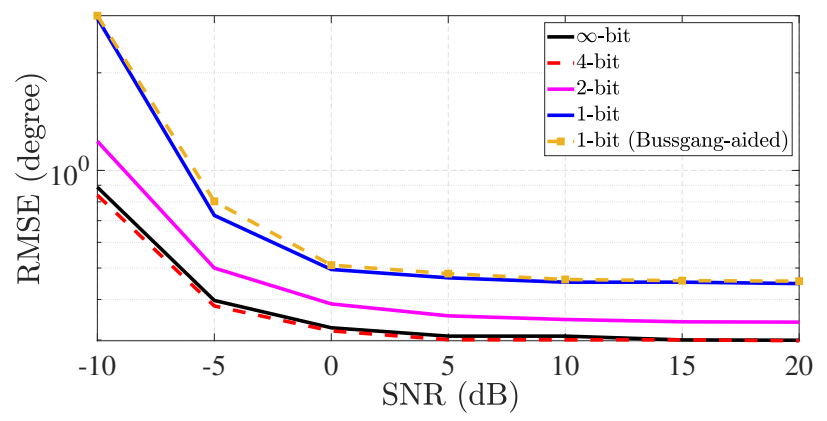

(b)

Figure 2. RMSE in degree for $\theta_{2}$ versus SNR for a nested array with $M=8$ elements and configuration given in (IV), $N=300$, and: (a) $K=4<M$; (b) $K=10>M$.

DoA estimation is very close to that of DoA estimates obtained from the unquantized array observations. For instance, when $K=4$, the performance loss arising from quantization, defined as $10 \log \left(\mathrm{RMSE}_{\text {quantized }} / \mathrm{RMSE}_{\text {unquantized }}\right)$, at $\mathrm{SNR}=5 \mathrm{~dB}$ are about 3.33 and $1.39 \mathrm{~dB}$ in case of 1-bit and 2-bit quantization, respectively, while it is almost zero in case of 4-bit quantization. However, the implementation costs and power consumption of 4-bit and 2-bit ADCs are still much lower compared to high-resolution ADCs. For example, at sampling frequency of $10 \mathrm{MHz}$, a 14-bit ADC consumes roughly $10^{3}$ times more power than 2-bit and 4-bit ADCs [35]. The gap between the power consumption of low- and high-resolution ADCs further increases with higher sampling frequencies, e.g. at sampling frequency of $1 \mathrm{GHz}$, a 14-bit ADC consumes roughly $10^{5}$ times more power than 2-bit and 4-bit ADCs [35]. Further, it is

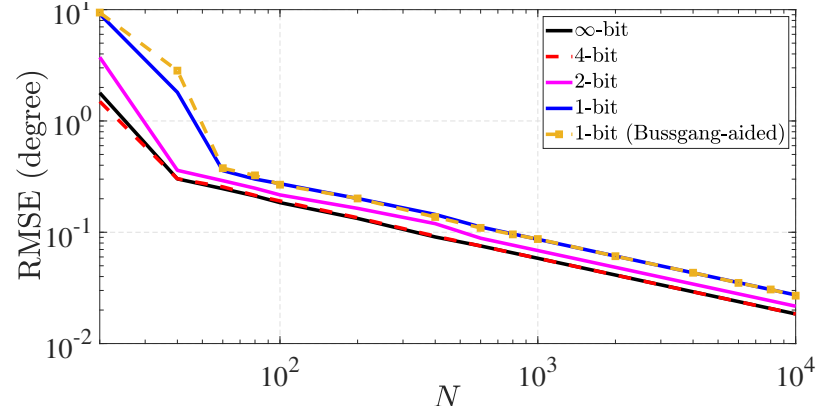

(a)

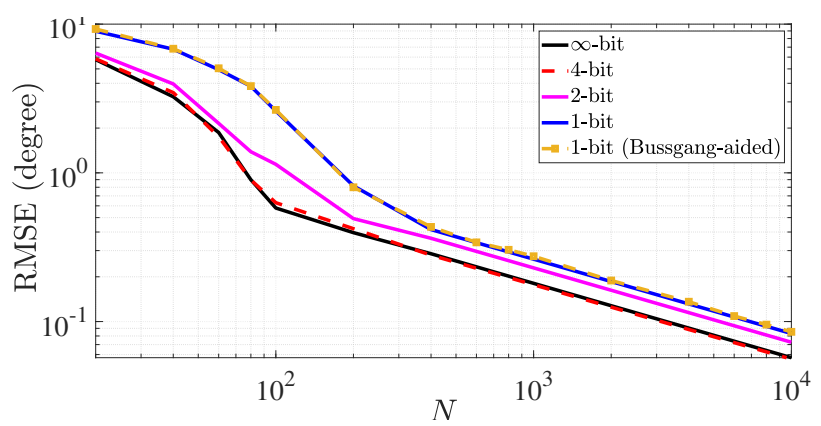

(b)

Figure 3. RMSE in degree for $\theta_{2}$ versus the number of snapshots for a nested array with $M=8$ elements and configuration given in (IV), SNR $=0 \mathrm{~dB}$, and: (a) $K=4<M$; (b) $K=10>M$.

relatively easy to implement 4-bit and 2-bit ADCs even at very high sampling frequencies while implementation feasibility of high-resolution ADCs moves from difficult at sampling frequencies of $\sim 1 \mathrm{MHz}$ to infeasible beyond those sampling frequencies [35]. Moreover, it is seen that the proposed method in case of 1-bit quantization performs as well as the one-bit DoA estimator in [24], which relies on estimating the covariance matrix of unquantized array observations directly from one-bit data using the Bussgang theorem.

Fig. 3 plots the RMSE for $\theta_{2}$ in degree versus the number of snapshots for SNR $=0 \mathrm{~dB}$ and: (a) $K=4<M$, and (b) $K=10>M$. Fig. 3 shows that, to achieve an RMSE of 0.1 for example, infinite-bit, 4-bit, 2-bit and one-bit cases need $300,300,500$ and 800 samples when $K=4$, respectively. This indicates that the total number of bits required to achieve an RMSE of 0.1 is, respectively, 1200, 1000 and 800 bits for 4-bit, 2-bit and one-bit sampling scenarios.

\section{CONCLUSiON}

The problem of DoA estimation from low-resolution fewbit SLA data was investigated. Firstly, the covariance matrix of unquantized array observations was retrieved from low-resolution few-bit SLA data by employing an iterative optimization-based algorithm. Then, DoAs were estimated by applying CAB-MUSIC to the recovered covariance matrix of unquantized array observations. The simulation results showed that increasing the sampling resolution to 2 or 4 bits per samples could significantly increase the DoA estimation performance compared to the one-bit sampling case while the power consumption and implementation costs are still much lower than the high-resolution sampling scenario. 


\section{REFERENCES}

[1] A. Paulraj, B. Ottersten, R. Roy, A. Swindlehurst, G. Xu, and T. Kailath, "subspace methods for directions-of-arrival estimation," Handbook of Statistics, vol. 10, pp. 693-739, 1993.

[2] F. Li, H. Liu, and R. J. Vaccaro, "Performance analysis for DoA estimation algorithms: unification, simplification, and observations," IEEE Trans. Aerosp. Electron. Syst, vol. 29, no. 4, pp. 1170-1184, Oct 1993.

[3] P. Stoica and A. Nehorai, "Performance study of conditional and unconditional direction-of-arrival estimation," IEEE Transactions on Acoustics, Speech, and Signal Processing, vol. 38, no. 10, pp. 17831795 , Oct 1990.

[4] S. S. Haykin, J. Litva, , and T. J. Shepherd, Eds., Radar Array Processing. Berlin, Germany: Springer-Verlag, 1993.

[5] A. Moffet, "Minimum-redundancy linear arrays," IEEE Transactions on Antennas and Propagation, vol. 16, no. 2, pp. 172-175, Mar 1968.

[6] P. P. Vaidyanathan and P. Pal, "Sparse sensing with co-prime samplers and arrays," IEEE Trans. Signal Process., vol. 59, no. 2, pp. 573-586, Feb 2011.

[7] P. Pal and P. P. Vaidyanathan, "Nested arrays: A novel approach to array processing with enhanced degrees of freedom," IEEE Transactions on Signal Processing, vol. 58, no. 8, pp. 4167-4181, Aug 2010.

[8] C. L. Liu and P. P. Vaidyanathan, "Cramér-Rao bounds for coprime and other sparse arrays, which find more sources than sensors," Digital Signal Processing, vol. 61, pp. 43 - 61, 2017.

[9] Y. D. Zhang, M. G. Amin, and B. Himed, "Sparsity-based DoA estimation using co-prime arrays," in 2013 IEEE International Conference on Acoustics, Speech and Signal Processing, May 2013, pp. 3967-3971.

[10] P. Pal and P. P. Vaidyanathan, "Pushing the limits of sparse support recovery using correlation information," IEEE Transactions on Signal Processing, vol. 63, no. 3, pp. 711-726, Feb 2015.

[11] Z. Tan and A. Nehorai, "Sparse direction of arrival estimation using co-prime arrays with off-grid targets," IEEE Signal Processing Letters, vol. 21, no. 1, pp. 26-29, Jan 2014.

[12] Z. Yang, L. Xie, and C. Zhang, "A discretization-free sparse and parametric approach for linear array signal processing," IEEE Transactions on Signal Processing, vol. 62, no. 19, pp. 4959-4973, Oct 2014.

[13] M. Wang and A. Nehorai, "Coarrays, MUSIC, and the Cramér-Rao bound," IEEE Trans. Signal Process., vol. 65, no. 4, pp. 933-946, Feb 2017.

[14] S. Sedighi, R. B. S. Mysore, S. Maleki, and B. Ottersten, "Consistent least squares estimator for co-array-based DoA estimation," in 2018 IEEE 10th Sensor Array and Multichannel Signal Processing Workshop (SAM), July 2018, pp. 524-528.

[15] S. Sedighi, B. S. M. R. Rao, and B. Ottersten, "An asymptotically efficient weighted least squares estimator for co-array-based DoA estimation," IEEE Transactions on Signal Processing, vol. 68, pp. 589-604, 2020.

[16] R. H. Walden, "Analog-to-digital converter survey and analysis," IEEE Journal on Selected Areas in Communications, vol. 17, no. 4, pp. 539-550, April 1999.

[17] X. Huang, S. Bi, and B. Liao, "Direction-of-arrival estimation based on quantized matrix recovery," IEEE Communications Letters, vol. 24 no. 2, pp. 349-353, 2020

[18] I. Yoffe, N. Regev, and D. Wulich, "On direction of arrival estimation with 1-bit quantizer," in 2019 IEEE Radar Conference (RadarConf), 2019, pp. 1-6.

[19] C. Stöckle, J. Munir, A. Mezghani, and J. A. Nossek, "1-bit direction of arrival estimation based on compressed sensing," in 2015 IEEE 16th International Workshop on Signal Processing Advances in Wireless Communications (SPAWC), June 2015, pp. 246-250.

[20] X. Huang, P. Xiao, and B. Liao, "One-bit direction of arrival estimation with an improved fixed-point continuation algorithm," in 2018 10th International Conference on Wireless Communications and Signal Processing (WCSP), 2018, pp. 1-4.

[21] X. Meng and J. Zhu, "A generalized sparse bayesian learning algorithm for 1-bit DoA estimation," IEEE Communications Letters, vol. 22, no. 7, pp. 1414-1417, 2018.

[22] T. Chen, M. Guo, and X. Huang, "Direction finding using compressive one-bit measurements," IEEE Access, vol. 6, pp. 41 201-41 211, 2018.

[23] X. Huang and B. Liao, "One-bit MUSIC," IEEE Signal Processing Letters, vol. 26, no. 7, pp. 961-965, July 2019.

[24] C. Liu and P. P. Vaidyanathan, "One-bit sparse array DoA estimation," in 2017 IEEE International Conference on Acoustics, Speech and Signal Processing (ICASSP), March 2017, pp. 3126-3130.

[25] K. N. Ramamohan, S. Prabhakar Chepuri, D. F. Comesaña, and G. Leus, "Blind calibration of sparse arrays for DoA estimation with analog and one-bit measurements," in ICASSP 2019 - 2019 IEEE International
Conference on Acoustics, Speech and Signal Processing (ICASSP), 2019, pp. $4185-4189$.

[26] Z. Cheng, S. Chen, Q. Shen, J. He, and Z. Liu, "Direction finding of electromagnetic sources on a sparse cross-dipole array using one-bit measurements," IEEE Access, vol. 8, pp. 83 131-83 143, 2020.

[27] C. Zhou, Y. Gu, Z. Shi, and M. Haardt, "Direction-of-arrival estimation for coprime arrays via coarray correlation reconstruction: A one-bit perspective," in 2020 IEEE 11th Sensor Array and Multichannel Signal Processing Workshop (SAM), 2020, pp. 1-4.

[28] O. Bar-Shalom and A. J. Weiss, "DoA estimation using one-bit quantized measurements," IEEE Transactions on Aerospace and Electronic Systems, vol. 38 , no. 3, pp. 868-884, July 2002

[29] M. Stein, K. Barbe, and J. A. Nossek, "DoA parameter estimation with 1-bit quantization bounds, methods and the exponential replacement," in WSA 2016; 20th International ITG Workshop on Smart Antennas, March 2016, pp. 1-6.

[30] S. Sedighi, M. R. B. Shankar, M. Soltanalian, and B. Ottersten, "On the performance of one-bit DoA estimation via sparse linear arrays," 2020. [Online]. Available: arXiv:2012.14051v1

[31] J. H. Van Vleck and D. Middleton, "The spectrum of clipped noise," Proceedings of the IEEE, vol. 54, no. 1, pp. 2-19, 1966.

[32] M. S. Gowda and R. Sznajder, "Schur complements, Schur determinantal and Haynsworth inertia formulas in Euclidean Jordan algebras," Linear Algebra Appl, vol. 432, pp. 1553-1559, 2010.

[33] R. A. Horn and C. R. Johnson, Matrix analysis. Cambridge university press, 2012.

[34] J. C. Bezdek and R. J. Hathaway, "Convergence of alternating optimization," Neural, Parallel \& Scientific Computations, vol. 11, no. 4, pp. 351-368, 2003

[35] F. Rivet, Y. Deval, J.-B. Begueret, D. Dallet, P. Cathelin, and D. Belot, "The experimental demonstration of a SASP-based full software radio receiver," IEEE Journal of Solid-State Circuits, vol. 45, no. 5, pp. 979988,2010 